\title{
High-sensitivity troponin assays in the evaluation of patients with acute chest pain in the emergency department
}

\author{
Michael Christ ${ }^{1}{ }^{1 *}$, Thomas Bertsch ${ }^{2}$, Steffen Popp ${ }^{1}$, \\ Philipp Bahrmann ${ }^{1}$, Hans-Jürgen Heppner ${ }^{1}$ and \\ Christian Müller ${ }^{3}$ \\ ${ }^{1}$ Department of Emergency and Critical Care Medicine, \\ City Hospital Nuremberg, Nuremberg, Germany \\ ${ }^{2}$ Institute of Laboratory Medicine, City Hospital \\ Nuremberg, Nuremberg, Germany \\ ${ }^{3}$ Department of Cardiology, University Hospital Basel, \\ Basel, Switzerland
}

\begin{abstract}
Evaluating patients with acute chest pain presenting to the emergency department remains an ongoing challenge. The spectrum of etiologies in acute chest pain ranges from minor disease entities to life-threatening diseases, such as pulmonary embolism, acute aortic dissection or acute myocardial infarction (MI). The diagnosis of acute MI is usually made integrating the triad of patient history and clinical presentation, readings of 12-lead ECG and measurement of cardiac troponins (cTn). Introduction of high-sensitivity cTn assays substantially increases sensitivity to identify patients with acute MI even at the time of presentation to the emergency department at the cost of specificity. However, the proportion of patients presenting with cTn positive, non-vascular cardiac chest pain triples with the implementation of new sensitive cTn assays increasing the difficulty for the emergency physician to identify those patients who are at need for invasive diagnostics. The main objectives of this mini-review are 1) to discuss elements of disposition decision made by the emergency physician for the evaluation of chest pain patients, 2) to summarize recent advances in assay technology and relate these findings into the clinical context, and 3 ) to discuss possible consequences for the clinical work and suggest an algorithm for the clinical evaluation of chest pain patients in the emergency department.
\end{abstract}

Keywords: acute chest pain; cardiac troponins; emergency department; high sensitivity cTn; myocardial infarction; risk stratification.

\footnotetext{
*Corresponding author: Prof. Dr. Michael Christ, Department of Emergency and Critical Care Medicine, Klinikum Nürnberg, Prof. Ernst Nathan Str. 1, 90419 Nürnberg, Germany

Phone: +49(911) 398 2369, Fax: +49(911) 398 3167,

E-mail: michael.christ@klinikum-nuernberg.de

Received March 9, 2011; accepted August 2, 2011;

previously published online September 6, 2011
}

\section{Introduction}

Evaluating patients with acute chest pain in the emergency department (ED) remains difficult despite an exponential growth of published research including innovations in diagnostic methods: Patients with acute coronary syndrome (ACS) including acute myocardial infarction (MI) should be effectively identified by the emergency physician in a timely manner to initiate specific clinical actions. Patients who do not have ACS or alternative high-risk situations should be safely discharged (1).

Triage decision in the ED is usually straightforward in patients presenting with typical cardiac chest pain and ST-segment elevation in the 12-lead ECG [Figure 1; (2)]. In contrast, disposition decision in patients with non-ST-elevation acute coronary syndrome [NSTE-ACS; $(3,4)$ ] is an ongoing challenge $(5,6)$ : The disposition decisions of the ED physician for patients with NSTE-ACS cannot be made by the use of time-dependent changes of cTn levels alone but are done by the integration of the triad of a) clinical presentation, b) readings of 12-lead ECG, and c) measurement of cardiac biomarkers, such as cTn, and/or MB-isoenzymes of creatine kinase and myoglobin at admission, and 6-12 h later [Figure 1; $(4,7)$ ]. The evaluation should include formal risk stratification using risk assessment tools, such as the TIMI, PURSUIT or GRACE risk scores $(8,9)$, because subjective risk assessment by emergency physicians appears cloudy due to the fact that important risk predictors, such as age and known coronary artery disease are often not adequately addressed (10). In particular situations, an earlier decision can be made by measuring cTn and ECG even 2 or $3 \mathrm{~h}$ after presentation to the ED to accelerate disposition decision $(11,12)$. Specific diagnostic work-up including computer tomography (CT) may be necessary for other differentials, such as suspected pulmonary embolism or aortic dissection. Using those various items for risk assessment, the ED physician has to decide whether the affected patient should be admitted to 1) an intervention (e.g., operating theatre, catheterization laboratory, or pharmacological interventions using thrombolysis), 2) the intensive care unit, 3) the coronary care unit, 4) a telemetry ward, 5) a hospital ward or 6) can be safely discharged into ambulatory care after non-invasive cardiac stress testing in the majority of cases.

Up to now, the practical consequences of the introduction of new, high-sensitivity assay technologies to measure cTn have not been adequately addressed for the work in the ED: cTn levels above the cut-off-limit alone with a rise or fall of concentration does not equal a diagnosis of MI. Non-vascular cardiac chest pain, such as acute heart failure due to hyper- 


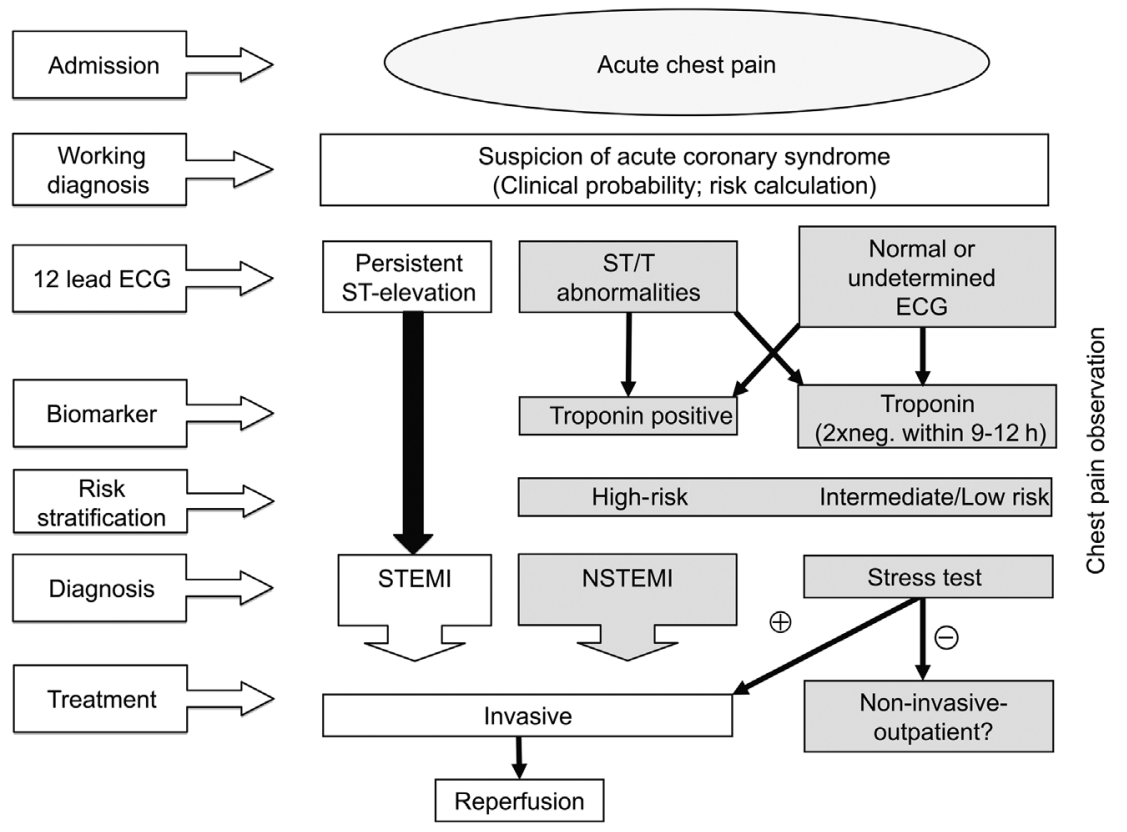

Figure 1 Suggested algorithm for decision-making in patients presenting with acute chest pain to the emergency department.

Triage decision is made using the triad of clinical signs and symptoms, results of 12-lead electrocardiogram and results of troponin testing. While ST-elevation myocardial infarction is straightforward, non-ST elevation acute coronary syndrome is challenging and affected patients need observation including follow-up 12-lead ECG and biomarker testing. Modified from (4).

tension may also be associated with rise and fall of cTn levels $(13,14)$. cTn is an extremely specific marker of cardiac injury $(15,16)$, however, acute myocardial damage is not specific to either MI or ACS.

The main objectives of this minireview are 1) to discuss elements of disposition decision made by the emergency physician for the evaluation of chest pain patients, 2) to summarize recent advances in assay technology and relate these findings into the clinical context, and 3) to discuss possible consequences for the clinical work and suggest an algorithm for the clinical evaluation of chest pain patients in the ED.

\section{Cardiac troponins: past}

The clinical definition of myocardial infarction includes a rise and/or fall of cardiac biomarkers (preferably cTn) with at least one value above the 99th percentile of the upper reference limit of a healthy reference population [Table 1; $(15,17)]$. Troponin $\mathrm{I}$ and $\mathrm{T}$ are structural proteins of the cardiomyocyte, and circulating levels of cTnI and cTnT can be measured using isoform-specific antibodies $(16,18)$. Increases of circulating cTnI or cTnT concentrations indicate cardiac muscle cell necrosis and are clinically used for the diagnosis of acute MI $(17,19)$. After the loss of integrity of cardiac cellular membranes, cardiac biomarkers diffuse into the cardiac interstitium, lymphatics, and microvasculature and can be measured in the peripheral circulation (20). The predominant portion of cTn (approx. 95\%) is bound via tropomyosin to actin filaments of the sarcomere, while a small proportion of $\mathrm{cTnI}(6 \%-8 \%)$ and $\mathrm{cTnT}(3 \%-8 \%)$ is probably located in the soluble cytoplasmic pool of the cardiac myocytes $(19,21)$. Considering methodological aspects of those experimental studies and the poor solubility of cTn in cytoplasm, it is probably better to use the term "early appearing pool" but not "cytoplasmic pool"' (16).

Cardiac troponins are currently considered the most sensitive and specific biomarkers of myocardial damage (4, 15-17, 22, 23). Increased levels of cTn levels can be meas-

Table 1 Definition of acute myocardial infarction as practically used in patients presenting with acute chest pain to the emergency department. Adapted from (13).

Detection of a rise and/or fall of cardiac biomarkers (preferably cTn) with at least one value above the 99th percentile of the upper reference limit of a healthy reference population and with at least one of the following:

Typical symptoms of cardiac ischemia

ECG changes indicative of new ischemia (new ST-T changes or new left bundle branch block)

Development of pathological Q-waves in the ECG

Imaging evidence of new loss of viable myocardium or a

Sudden, unexpected cardiac death, involving cardiac arrest, often with symptoms suggestive of myocardial ischemia, and accompanied by presumably new ST elevation or new left bundle branch block, and/or evidence of fresh thrombus by coronary angiography and/or autopsy 
ured during occlusion of large epicardial coronary arteries, increased myocardial strain, sepsis, exposure to toxins, inflammation or trauma (13). Dynamic changes of cTn release after myocardial damage depends on the intracellular compartmentalization of cTn: Irreversible damage of a cardiac myocytes induces immediate release of the "early appearing pool" of cTn followed by a more continuous release of the myofibril-bound troponins (20). cTn levels usually peak at 12-24 h after onset of chest pain and may remain abnormal up to 2 weeks in large MI $(20,24)$. Small increases of cTn, which may be due to plaque rupture resulting in microinfarction or non-coronary reasons of myocyte necrosis, such as in acute heart failure, sepsis or tachycardia, may be related to the release of cTn from the early appearing pool of cTn due to cell membrane instability induced by ischemia, wall strain or toxins (13).

During the introduction of cTn assays in routine practice in the late 1990s, evaluation of patients with acute MI revealed that cTn assays are more sensitive for small areas of MI than those for CK-MB assays resulting in confusing situations due to the used definition of MI at that time (25). The precision of those former cTn assays was low. Since cTn assays have been validated by the use of the former WHO definition of acute MI using CK-MB assays as gold standard (25), increased cTn levels have been seen in ACS patients with CK-MB levels within the normal reference limits. Also of interest, increased levels of circulating cTn without accompanying increases of CK-MB are associated with increased risk of death, and acute MI. Even small increases of cTn levels support to identify ACS patients at increased risk, who benefit from early invasive therapy $(1,4)$.

The high specificity of increased cTn levels for myocardial damage measured by current assay technology have been acknowledged by recent guidelines $(3,4,26)$ and routine evaluation of chest pain patients using biologic markers, such as CK-MB or myoglobin have been questioned (15). Measurement of CK-MB may have some value in the interpretation of reinfarction in patients with already increased cTn levels due to recent MI or in patients with increased cTn levels due to renal dysfunction. Before changing the practice of initial assessment of chest pain patient in the ED, it is prudent to check the used laboratory methods of cTn measurement. Different approaches than those suggested above may be applied with the use of point-of-care cTn measurements, which often display a lower sensitivity than modern high-sensitivity cTn assays used in clinical laboratory (27).

\section{Cardiac troponins: present}

During recent years, cTn assay technology has been considerably improved including improvements of analytical sensitivity and precision $(23,28)$. cTn assays, which are able to measure circulating cTn levels in more than $50 \%$ of a normal reference population, have been suggested to be called "high-sensitive"' cTn assays (29). In the meantime, cTn levels can even be measured in $>80 \%$ of healthy people with a coefficient of variation $<10 \%$ at the recommended cutpoint $(18,30)$. High-sensitivity assays are 1000-10,000-fold more sensitive than the original first generation assays due to improvement of epitope selection of antibodies and innovative capture and detection techniques (1).

The analytical sensitivity and high precision of sensitive cTn assay technology enables to lower the cTn cut-off-point for diagnosis of acute MI with accordance to the current definitions (cutpoint defined as the 99th percentile of the upper reference limit of a healthy reference population (16, 17); Figure 2). The use of a population-defined upper limit of normality at the 99th percentile results in a $160 \%$ increase of chest pain patients diagnosed with acute MI and a $200 \%$ increase of cTn positive patients with non-coronary cardiac chest pain (14). Additionally, more patients with acute MI display increased hs-cTn levels even at the time of presentation at the $\operatorname{ED}(11,14,31-33)$.

Due to the sensitivity and specificity of cardiac troponins for detecting myocardial damage, measurement of cTn is the ideal biomarker to diagnose acute MI, perform risk stratification in affected patients and initiate specific clinical action (34). Subsequently, cTnI or cTnT are the biologic markers of choice in the evaluation of patients with chest pain (15, 16). However, the introduction of these new, high-sensitivity assays to measure cTnT or cTnI may have several implications for clinical practice.

1. The definition of the cutpoint at the 99th percentile of a healthy reference population is derived from an epidemiological standpoint. The clinical impact of this definition is currently not well characterized although studies indicate that even small elevations of cTn levels indicate worse prognosis (35-37). In addition, there is currently no standardization of a reference population, which leads to variations of the 99th percentile by demographics and according to the screening methods used to exclude significant cardiac disease: More intense screening of cardiovascular disease reduced the 99th percentile of cTnI from $44 \mathrm{ng} / \mathrm{L}$ to $28 \mathrm{ng} / \mathrm{L}$ in the Perspective Study of the Vasculature in Uppsala Seniors (38). Additionally, there is no consensus on the use of age-specific reference intervals and gender dependent cutpoints for cTn.

2. Using high-sensitivity assays, low amounts of cTn released from cardiomyocytes during strenuous exercise or tachycardia can be measured in apparently healthy people even in the absence of irreversible myocardial damage (13). This may occur during benign tachyarrhythmic events, such as in atrial tachyarrhythmia, in AV-node reentrant tachycardia exemplary or during strenuous exercise as summarized by Michielsen et al. (39). It is currently not clarified whether irreversible damage of myocardial cells or a transient leakage of cTn from the early appearing pool is responsible for this observation $(13,18)$. Delayed-enhancement cardiac magnetic resonance imaging in a marathon study supports the release from the "early appearing pool" without irreversible damage of myocytes. Increased membrane permeability has been suggested to be a likelier mechanism than myocardial necrosis for exercise-induced cTn release $(13,40)$. These highly sensitive cTn assays may lead to an increased proportion of patients with cTn levels above the cutpoint and may increase the admission rate of 


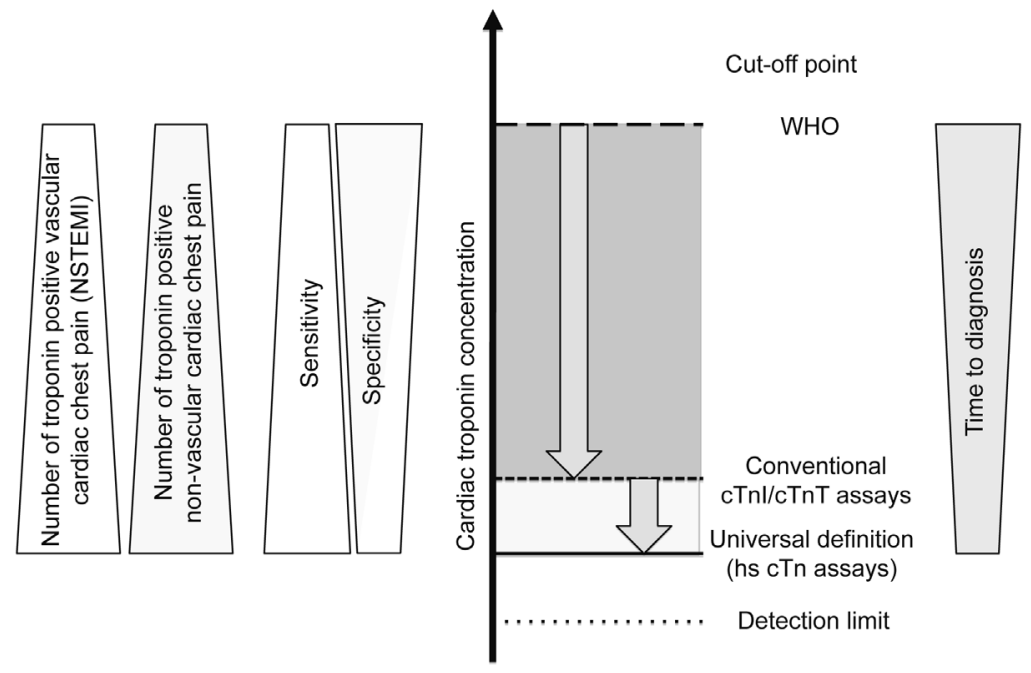

Figure 2 Schematic outline of changes resulting from the lowering of cut-off concentrations of cardiac troponin (cTn) levels in patients presenting with chest pain to the emergency department.

Originally, cut-off concentration was set according to the definition of acute myocardial infarction by the World Health Organization (WHO). Universal definition of acute myocardial infarction includes an increase of cTn levels above the 99\% percentile of a healthy reference population at a coefficient of variation $<10 \%$ (17). While cut-off-levels of conventional cTn assays could not achieve the limit at the $99 \%$ percentile of the reference population due to high variation of results at low cTn concentrations, new, high-sensitivity cTn assays are able to measure circulating cTn levels in healthy persons at high precision. Lowering of cut-off-points increase sensitivity and the number of chest pain patients with increased cTn at the cost of specificity. Increased cTn levels are found in almost all patients with acute myocardial even at the time of presentation at the emergency department $(14,32,33,55)$.

affected patients and the number of resources needed to exclude cardiac dysfunction. Our own experience showed that emergency physicians can handle these challenges without difficulties: the admission rate has not changed after implementation of the new assay technology (14).

3. Levels of cTn as measured with the new, high-sensitivity assays are associated with elevated cardiovascular risk in apparently healthy, middle aged men (30). Few study subjects in this cohort display cTn levels above the recommended new cutpoint for the diagnosis of acute MI (30). Moreover, even small increases of cTnI levels in patients presenting with acute chest pain to the ED are indicative for adverse short- and long-term prognosis $(35,37)$. Also of interest, chest pain patients with small increases of cTn levels, which have been not identified using former assay generations, may also benefit from early coronary intervention (36).

Circulating cTn are associated with the extent of coronary artherosclerosis meaning higher plaque burden in subjects with higher cTn levels (41). Higher cTn levels are associated with increased overall mortality in affected patients with stable coronary artery disease (42). Of note, increased cTn concentrations in the latter cohort of apparently healthy men were associated with an increased incidence of cardiovascular death and heart failure but not with an increased rate of acute MI (42). It is currently unknown whether preventive actions may beneficially influence this minor, but increased risk of adverse events.

Summing up, methodological aspects of current cTn assays have been considerably improved and cTn levels can be measured even in healthy people. We need to reassess the currently held belief that cTn is released only from irreversibly injured myocardial cells and indicated acute MI (13). Nevertheless, even small increases of cTn levels in patients with coronary artery disease or heart failure indicate increased risk of adverse outcome and may be due to various conditions. The difficulty for the emergency physician is in deciding how to use this high quality and previously unobtainable information (1). It may not be clear whether the new high-sensitivity cTnI and cTnT methods will lead to more clarity or confusion in routine clinical practice (28). It is currently discussed that the time-dependent rise and fall (delta-change) of cTn levels within a few hours of observation may be supportive for the emergency physician to diagnose of acute MI.

\section{Time-dependent changes of troponin levels in acute myocardial infarction}

Increased analytical cTn assay sensitivity is associated with an elevated rate of patients with increased cTn levels presenting to the ED additionally detecting pathological processes of the heart other than acute ischemia and/or acute MI (14, 33). Formally, a time-dependent rising and falling pattern of cTn levels is included in the definition of acute MI (17). Due to analytical variability, a $20 \%$ time-dependent change of cTn levels has been suggested to be clinically meaningful (43). Giannitsis et al. analyzed chest pain patients with negative cTnT levels at initial presentation and confirmed NSTEMI using the 4th generation cTnT assay for adjudicated diagnosis of acute MI. They found that patients 
with acute MI display a $>100 \%$ and $>200 \%$ change of hs-cTnT levels within 3 and $6 \mathrm{~h}$ after initial presentation. Using those time-dependent delta-change criterium for the diagnosis of acute MI a $100 \%$ positive predictive value is obtained (44). It is interesting that in most studies examining the variability of hs-cTnT and hs-cTnI levels in healthy study subjects, an overall variability $>50 \%$ has been shown within a few hours of observation (45-48). These findings confirm the suggestion that a time-dependent change of hs-cTnI or hs-cTnT levels $>50 \%$ may be useful as a important diagnostic criterium for the diagnosis of acute $\mathrm{MI}$ in the ED. Aldous et al. examined the introduction of the suggested time-dependent delta-change criterium for the diagnosis of acute MI in the ED (49): Introduction of the diagnostic criterium that a time-dependent increase (or fall) of hs-cTn $>50 \%$ is necessary to diagnose acute $\mathrm{MI}$ increase specificity $>95 \%$ at the cost of sensitivity (hs-cTnT: $61.8 \%$; hs-cTnI: $68.2 \%)$. These findings support the notion that diagnostic reasoning in emergency medicine cannot be reduced to pure mathematical calculations of biomarker concentrations (49, 50). Diagnostic reasoning and deduced therapeutic management strategies can only be made correctly, when all available information about the patient is used. This includes intuitive and analytical approaches (51) and should consider prevailing discomfort of the patient, clinical signs and symptoms at examination, results of 12-lead electrocardiogram, and levels and dynamics of cTn levels to calculate post-test probabilities (50). Moreover, the task of the emergency physician is not limited to rule in or rule out acute MI in the $\mathrm{ED}$, but to find the correct triage decision. Emergency physicians have to identify patients at increased risk for adverse outcome, who may benefit from hospitalization and/or tailored treatment strategies.

\section{Clinical decision-making in chest pain patients}

The recently released document on the "universal definition of MI" has strengthened the role of cTn increases as an important criterion for the diagnosis of MI (17). Subsequently, the emphasis on biomarkers, such as cTn in clinical decision-making of chest pain patients in ED has increased (15). However, overreliance on $\mathrm{cTn}$ measurements and failure to consider clinical presentation and ECG findings can lead to over- and underdiagnosis of MI, each of which carries its own set of hazards $(50,52)$.

Cardiac troponins are useful not only because they come close to fulfilling many of the criteria for an ideal biomarker, but also because they convey prognostic information and can help frame therapeutic decisions regarding patients with acute coronary syndrome. Elevated cTn levels in acute NSTE-ACS indicates adverse prognosis and coronary intervention appears prudent $(3,4,36)$. Note that the introduction of risk stratification tools using clinical information as the GRACE risk model may help to identify patients with increased cTn levels, who benefit from revascularization (53). Thus, increases of cTn levels combined with clinical information indicate the need for a specific treatment strategy, which has been shown to be associated with a significant reduction of death or recurrent MI during follow-up (54). A recently published study indicates that chest pain patients, who are newly identified using sensitive cTn assay technology, are at increased risk and may benefit from early revascularization (36). However, because these highly precise and sensitive laboratory assays have not been formally tested with currently used algorithms (Figure 1), clinical decisionmaking for patients presenting with chest pain to the $\mathrm{ED}$ using high-sensitivity cTn assay may change.

Reichlin et al. report results of a multicenter study recruiting 718 consecutive patients who presented with symptoms suggesting acute coronary syndrome in the ED (33). Using the 4th generation cTnT assay and all clinical information obtained during the hospital stay for adjudicated final diagnosis of acute MI, $17 \%$ of patients of the cohort had acute MI. Highly sensitive cTnT and cTnI assays at presentation displayed a significant higher diagnostic accuracy as measured by the area under receiver-operating-characteristics curve (AUC) than the standard assay [standard test: 0.90 vs. sensitive assays: $0.92-0.98$; (33)]. Sensitivity of high-precision methods to detect acute MI at presentation ranged from $85 \%-95 \%$ compared to $72 \%$ using the conventional cTn assay. However, specificity significantly decreased with the new method compared to the conventional assay ( $80 \%$ vs. 97\%). Comparable results were reported in a prospective multicenter study of patients with chest pain and even small increases of sensitive cTnI levels were associated with increased risk of adverse events within 30 days of follow-up (32). Several studies confirm the results that high sensitive cTn assays identify patients with acute MI earlier than less sensitive assays $(11,44,55)$. Introduction of the new method in routine practice will increase the number of positive test results without changing the number of tests $(55,56)$, while the number of cardiologist consultations increased possibly due to the challenge in patients with consistently low-positive results without dynamic changes during follow-up (31).

Recently, we examined the impact of introducing a sensitive cTn test into routine clinical practice (14), 137 consecutive patients presenting with acute chest pain were examined using the 4th generation cTnT assay and the new, high-sensitivity cTnT assay for the definition of acute MI. Sensitivity to detect a patient presenting with acute MI at presentation was significantly lower with the conventional hs-cTnT compared to the sensitive cTn assay (57\% vs. 94.3\%). Negative predictive value was higher for hs-cTnT (97.3\%) compared to cTnT (78.6\%). Using current cTnT assays, $7 \%$ of chest pain patients presenting to the ED display cTnT positive, non-vascular cardiac chest pain and the proportion of patients presenting with chest pain and abnormal hs-cTnT concentrations nearly tripled using the new assay. The number of patients with unstable angina or cardiac non-vascular chest pain without $\mathrm{cTn}$ elevation significantly decreased. Patients with acute MI display a significant rise or fall of hs-cTnT levels within a few hours, while time-dependent dynamics of hs-cTnT changes were modest in patients with non-vascular cardiac chest pain. Even modest increases of cTn in this population indicate increased risk of death or recurrent MI within 6 months of follow-up, while risk was low in patients with hs-cTnT levels $<14 \mathrm{ng} / \mathrm{L}$. It 
was interesting that the risk for an event was highest in chest pain patients with dynamic changes of hs-cTnT levels $>30 \%$ (Figure 3).

Elevated cTn levels in patients with sepsis, hypertensive emergency, pulmonary embolism, hypotension, renal failure, or any of several other conditions indicates that myocardial damage or even non-thrombotic MI has occurred, producing leakage of cTn. In this situation, the underlying reason of the disease has to be treated and increased levels of sensitive cTn possibly indicate worse prognosis but will not change the management of affected patients (13). Thus, small increases of cTn levels in patients presenting with chest pain have to be judged with prudence and adjudication of disposition decision should include general risk assessment (50).

In conclusion, introduction of high-sensitivity cTn assays increases the sensitivity to identify patients with acute MI even at the time of initial presentation in the ED at the cost of specificity. Several studies indicate that even small increases of cTn levels are clinically relevant. Some investigators propose that the specificity of high sensitive assays to diagnose acute MI may be improved using a defined timedependent delta-change criterium [see above, $(44,57)]$. However, introduction of those criteria may again decrease sensitivity to diagnose acute MI and should therefore be used only within the clinical context. Correct interpretation of the low-positive cTn results is important for development of algorithms that help to triage affected patients without overor underuse of consultation or invasive treatment.

\section{How to use high-sensitive cTn assays in clinical practice?}

New, high-sensitivity cTn assays display several advantages for patients presenting with acute chest pain to the ED, such as potential for earlier detection of acute MI and greater sensitivity in identifying acute myocardial injury. However, interpretation of results in a distinct patient with acute chest pain has to be readjusted and a significant rise and/or fall of cTn levels during serial testing should be included to diagnose acute MI (50). Even with conventional cTn assays, patients can have detectable cTn levels because of etiologies other than vascular occlusion and the use of high-sensitive cTnI or cTnT doubles such "false-positive" findings (14). In the past, lessons interpreting results obtained with new methods in the diagnosis of acute MI and resulting need for coronary intervention have been learned after the introduction of cTn assays in the end of the 1990s (58) and after the updated definition of acute MI 2000 (59) followed by improved assay methodology during recent years [Figure 3; (16)].

What are the key points for the work with high-sensitive cTn assays in the ED?

First of all, the ED physician should keep in mind that an increased high-sensitivity cTn level does not equal the diagnosis of acute MI and therefore should not necessarily lead to ACS-directed care. However, increased cTn levels indicate adverse outcome, while prognosis of patients with cTn levels below the 99th percentile cutpoint is favorable. Of note, cTn levels below the cut-off-point even during follow-up do not exclude the necessity of further work-up of troponin-negative ACS, although cTn levels below the cut-off-point indicate beneficial prognosis (14).

Second, ED diagnosis of acute MI consists of the correct interpretation of the triad of I) typical clinical presentation, II) interpretation of the 12-lead ECG, and III) increased levels of cTn with a rising and falling pattern of cTn during follow-up.

Dynamic changes of cTn during follow-up do not indicate acute MI due to coronary obstruction, but rather an acute myocardial injury (e.g., MI, sepsis, hypertensive emergency, pulmonary embolism, acute heart failure or tachyarrhythmia). Clinical probability of ACS is essential to make the correct triage decision $(49,50,60)$. Recently, a formal evaluation of pretest probability has been performed using TIMI risk score and further patient disposition was done after determination of post-test probability using a cardiac multimarker assay. Using this approach, safe patient discharge could be reduced to $2 \mathrm{~h}$ (12). Additional use of non-invasive imaging or stress testing may support the request for a correct decision in the ED.

Third, new high-sensitivity cTn assays are able to detect levels of cTn that would register as zero with conventional assays $(14,32,33,55)$. Thus, acute myocardial damage will be detected earlier than with the use of former assays. Specifically, patients presenting within $3 \mathrm{~h}$ after onset of chest pain may have raised cTn levels (33). Those data and our own experience indicate that serial cTn testing may be shortened to 2 or $3 \mathrm{~h}$ after initial presentation of chest pain patients to the emergency department (11). However, prospective data of how to use new cTn measurement are currently rare and a follow-up of patients at risk with follow-up of 12-lead ECG and cTn determination should be guaranteed until further data are available (9-12 h after onset of symptoms or 6-9 $\mathrm{h}$ after presentation to the ED).

Fourth, we need to reassess the current hypothesis that cTn is released only from irreversibly injured myocardial cells. Even apparently healthy subjects with reentrant tachycardia or after strenuous exercise will display increased levels of high-sensitive cTn with a rise and falling pattern during follow-up. However, absolute concentrations of cTn levels are usually low. Correct disposition decision depends on the correct interpretation of pretest probabilities as discussed above $(8,60,61)$. The use of a defined height of dynamic changes of cTn levels during follow-up as suggested $(44,57)$ may not adequately address this challenge, since specificity may decrease and even small increases of cTn may indicate microinfarction coexistent with plaque rupture and even troponin-negativity may harbor the risk for adverse events (28, 49). Possibly, clinical decision aids will help to overcome these problems (5). However, some of these aids have been tested in the pre-troponin era questioning their validity with the use of new sensitive assays and a recent metanalysis suggests that clinical decision aids may be helpful but will not substitute the experience of an emergency physician (62, 63). Furthermore, technical advances of revascularization have been considerably improved in the meantime changing 

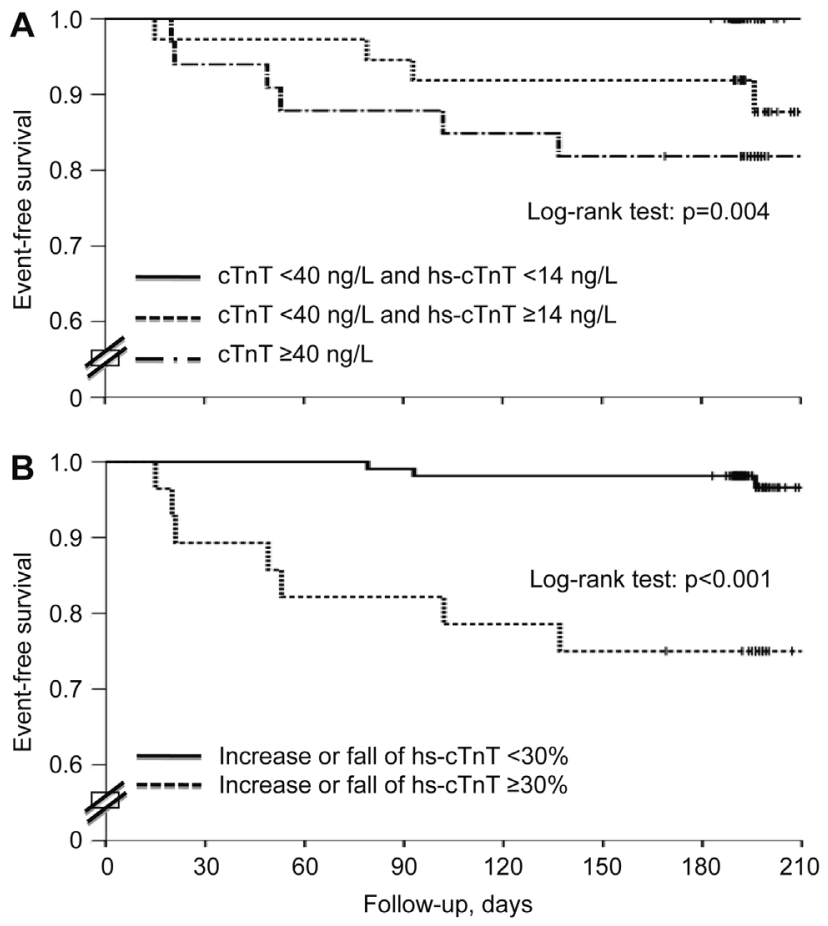

Figure 3 Kaplan-Meier analysis of patients presenting with acute chest pain to the emergency department.

The event rate (combined endpoint: death or recurrent myocardial infarction during 7 months of follow-up) was low in patients with hs-cTnT levels $<14 \mathrm{ng} / \mathrm{L}$, while patients with cTnT levels $\geq 40 \mathrm{ng} / \mathrm{L}$ were at an increased risk for the combined endpoint. Patients with hs-cTnT levels $\geq 14 \mathrm{ng} / \mathrm{L}$ and cTnT levels $<40 \mathrm{ng} / \mathrm{L}$ had an intermediate risk (A). Risk for the combined endpoint was highest in patients with a rise or fall of hs-cTnT levels $>30 \%$ within the first $24 \mathrm{~h}$ after presentation (B). Vertical lines indicate censoring of data. Modified from (14).

the management of affected patients compared to treatment in the past. For decision- and disposition-making in the ED, we use the algorithm recently suggested, which includes individual risk assessment by an experienced emergency physician [Figure 1;(4)]. Collinson asked whether troponins are confusing, and answered "not really, unless you stop thinking clinically" (64), which is true more than ever.

\section{Conclusions}

New, high-sensitivity cTn assays will enable physicians to both confirm and exclude MI sooner than is now possible in patients presenting with acute chest pain to the ED, but serial cTn testing remains necessary. With the increased sensitivity of new cTn assays, the proportion of patients with cTn positive, non-vascular cardiac chest pain may increase substantially. Therefore, electrocardiographic and clinical probability of acute coronary syndrome become more, not less important, in order to come to the correct triage decision in the ED (50). cTnI or cTnT levels below the 99th percentile cutpoint and lack of a rise and falling pattern of cTnI/cTnT levels during follow-up indicates beneficial prognosis, but do not exclude the diagnosis of relevant coronary artery disease. Failure to consider clinical presentation and ECG findings appropriately, and overreliance on cTn measurement may lead to both, over- and underdiagnosis of acute coronary syn- drome including MI or unstable angina, each of which carries its own hazards (52).

\section{Conflict of interest statement}

Authors' conflict of interest disclosure: The authors stated that there are no conflicts of interest regarding the publication of this article. Research granting played no role in the study design; in the collection, analysis, and interpretation of data; in the writing of the report; or in the decision to submit the report for publication.

Research funding: None declared.

Employment or leadership: None declared.

Honorarium: None declared.

Disclosure: MC and TB have received grants and speaking honoraria from Roche AG, Germany. CM has received grants and speaking honoraria from Roche AG, Siemens AG, and Abbott, Germany.

\section{References}

1. Baker JO, Reinhold J, Redwood S, Marber MS. Troponins: redefining their limits. Heart 2011;97:447-52.

2. Antman EM, Anbe DT, Armstrong PW, Bates ER, Green LA, Hand $\mathrm{M}$, et al. ACC/AHA guidelines for the management of patients with ST-elevation myocardial infarction: a report of the American College of Cardiology/American Heart Association Task Force on Practice Guidelines (Committee to Revise the 
1999 Guidelines for the Management of Patients with Acute Myocardial Infarction). Circulation 2004;110:e82-292.

3. Anderson JL, Adams CD, Antman EM, Bridges CR, Califf RM, Casey DE, Jr., et al. ACC/AHA 2007 guidelines for the management of patients with unstable angina/non ST-elevation myocardial infarction: a report of the American College of Cardiology/American Heart Association Task Force on Practice Guidelines (Writing Committee to Revise the 2002 Guidelines for the Management of Patients With Unstable Angina/Non ST-Elevation Myocardial Infarction): developed in collaboration with the American College of Emergency Physicians, the Society for Cardiovascular Angiography and Interventions, and the Society of Thoracic Surgeons: endorsed by the American Association of Cardiovascular and Pulmonary Rehabilitation and the Society for Academic Emergency Medicine. Circulation 2007;116:e148-304.

4. Bassand JP, Hamm CW, Ardissino D, Boersma E, Budaj A, Fernandez-Aviles F, et al. Guidelines for the diagnosis and treatment of non-ST-segment elevation acute coronary syndromes. Eur Heart J 2007;28:1598-660.

5. Lee TH, Goldman L. Evaluation of the patient with acute chest pain. N Engl J Med 2000;342:1187-95.

6. Scirica BM. Acute coronary syndrome: emerging tools for diagnosis and risk assessment. J Am Coll Cardiol 2010;55:140315 .

7. Fesmire FM, Decker WW, Diercks DB, Ghaemmaghami CA, Nazarian D, Brady WJ, et al. Clinical policy: critical issues in the evaluation and management of adult patients with non-STsegment elevation acute coronary syndromes. Ann Emerg Med 2006;48:270-301.

8. Body R. Acute MI: triple-markers resurrected or Bayesian dice? Lancet 2011;377:1049-50.

9. Kontos MC, Diercks DB, Kirk JD. Emergency department and office-based evaluation of patients with chest pain. Mayo Clin Proc 2010;85:284-99.

10. Yan AT, Yan RT, Huynh T, Casanova A, Raimondo FE, Fitchett $\mathrm{DH}$, et al. Understanding physicians' risk stratification of acute coronary syndromes: insights from the canadian ACS 2 registry. Arch Intern Med 2009;169:372-8.

11. Scharnhorst V, Krasznai K, van't Veer M, Michels R. Rapid detection of myocardial infarction with a sensitive troponin test. Am J Clin Pathol 2011;135:424-8.

12. Than M, Cullen L, Reid CM, Lim SH, Aldous S, Ardagh MW, et al. A 2-h diagnostic protocol to assess patients with chest pain symptoms in the Asia-Pacific region (ASPECT): a prospective observational validation study. Lancet 2011;377: 1077-84.

13. Agewall S, Giannitsis E, Jernberg T, Katus H. Troponin elevation in coronary vs. non-coronary disease. Eur Heart J 2011; 32:404-11.

14. Christ M, Popp S, Pohlmann H, Poravas M, Umarov D, Bach $\mathrm{R}$, et al. Implementation of high sensitivity cardiac troponin $\mathrm{T}$ measurement in the emergency department. Am J Med 2010;123:1134-42.

15. Saenger AK, Jaffe AS. Requiem for a heavyweight: the demise of creatine kinase-MB. Circulation 2008;118:2200-6.

16. Thygesen K, Mair J, Katus H, Plebani M, Venge P, Collinson $\mathrm{P}$, et al. Recommendations for the use of cardiac troponin measurement in acute cardiac care. Eur Heart J 2010;31:2197-204.

17. Thygesen K, Alpert JS, White HD. Universal definition of myocardial infarction. Eur Heart J 2007;28:2525-38.

18. Giannoni A, Giovannini S, Clerico A. Measurement of circulating concentrations of cardiac troponin $\mathrm{I}$ and $\mathrm{T}$ in healthy subjects: a tool for monitoring myocardial tissue renewal? Clin Chem Lab Med 2009;47:1167-77.

19. Katus HA, Remppis A, Scheffold T, Diederich KW, Kuebler W. Intracellular compartmentation of cardiac troponin $\mathrm{T}$ and its release kinetics in patients with reperfused and nonreperfused myocardial infarction. Am J Cardiol 1991;67:1360-7.

20. Antman EM, Tanasijevic MJ, Thompson B, Schactman M, McCabe $\mathrm{CH}$, Cannon $\mathrm{CP}$, et al. Cardiac-specific troponin I levels to predict the risk of mortality in patients with acute coronary syndromes. N Engl J Med 1996;335:1342-9.

21. Bleier J, Vorderwinkler KP, Falkensammer J, Mair P, Dapunt $\mathrm{O}$, Puschendorf B, et al. Different intracellular compartmentations of cardiac troponins and myosin heavy chains: a causal connection to their different early release after myocardial damage. Clin Chem 1998;44:1912-8.

22. Apple FS, Wu AH, Jaffe AS, Panteghini M, Christenson RH. National Academy of Clinical Biochemistry and IFCC Committee for Standardization of Markers of Cardiac Damage Laboratory Medicine Practice Guidelines: analytical issues for biomarkers of heart failure. Clin Biochem 2008;41:222-6.

23. Plebani M, Zaninotto M. Cardiac troponins: what we knew, what we know - where are we now? Clin Chem Lab Med 2009; 47:1165-6.

24. Morrow DA, Cannon CP, Jesse RL, Newby LK, Ravkilde J, Storrow AB, et al. National Academy of Clinical Biochemistry Laboratory Medicine Practice Guidelines: Clinical Characteristics and utilization of biochemical markers in acute coronary syndromes. Circulation 2007;115:e356-75.

25. Wu AH, Apple FS, Gibler WB, Jesse RL, Warshaw MM, Valdes R, Jr. National Academy of Clinical Biochemistry Standards of Laboratory Practice: recommendations for the use of cardiac markers in coronary artery diseases. Clin Chem 1999; 45:1104-21.

26. Apple FS, Jesse RL, Newby LK, Wu AH, Christenson RH. National Academy of Clinical Biochemistry and IFCC Committee for Standardization of Markers of Cardiac Damage Laboratory Medicine Practice Guidelines: analytical issues for biochemical markers of acute coronary syndromes. Circulation 2007;115:e352-5.

27. Storrow AB, Lyon JA, Porter MW, Zhou C, Han JH, Lindsell CJ. A systematic review of emergency department point-of-care cardiac markers and efficiency measures. Point of Care 2009;8:121.

28. White HD. Will new higher-precision troponins lead to clarity or confusion? Curr Opin Cardiol 2008;23:292-5.

29. Apple FS. A new season for cardiac troponin assays: it's time to keep a scorecard. Clin Chem 2009;55:1303-6.

30. Otsuka T, Kawada T, Ibuki C, Seino Y. Association between high-sensitivity cardiac troponin $\mathrm{T}$ levels and the predicted cardiovascular risk in middle-aged men without overt cardiovascular disease. Am Heart J 2010;159:972-8.

31. Melanson SE, Conrad MJ, Mosammaparast N, Jarolim P. Implementation of a highly sensitive cardiac troponin I assay: test volumes, positivity rates and interpretation of results. Clin Chim Acta 2008;395:57-61.

32. Keller T, Zeller T, Peetz D, Tzikas S, Roth A, Czyz E, et al. Sensitive troponin I assay in early diagnosis of acute myocardial infarction. N Engl J Med 2009;361:868-77.

33. Reichlin T, Hochholzer W, Bassetti S, Steuer S, Stelzig C, Hartwiger $\mathrm{S}$, et al. Early diagnosis of myocardial infarction with sensitive cardiac troponin assays. N Engl J Med 2009;361: 858-67.

34. Morrow DA, de Lemos JA. Benchmarks for the assessment of novel cardiovascular biomarkers. Circulation 2007;115:94952. 
35. Kavsak PA, Wang X, Ko DT, MacRae AR, Jaffe AS. Short- and long-term risk stratification using a next-generation, high-sensitivity research cardiac troponin I (hs-cTnI) assay in an emergency department chest pain population. Clin Chem 2009;55:1809-15.

36. Mills NL, Churchhouse AM, Lee KK, Anand A, Gamble D, Shah AS, et al. Implementation of a sensitive troponin I assay and risk of recurrent myocardial infarction and death in patients with suspected acute coronary syndrome. J Am Med Assoc 2011;305:1210-6.

37. Bonaca M, Scirica B, Sabatine M, Dalby A, Spinar J, Murphy SA, et al. Prospective evaluation of the prognostic implications of improved assay performance with a sensitive assay for cardiac troponin I. J Am Coll Cardiol 2010;55:2118-24.

38. Eggers KM, Jaffe AS, Lind L, Venge P, Lindahl B. Value of cardiac troponin I cutoff concentrations below the 99th percentile for clinical decision-making. Clin Chem 2009;55:85-92.

39. Michielsen EC, Wodzig WK, Van Dieijen-Visser MP. Cardiac troponin $\mathrm{T}$ release after prolonged strenuous exercise. Sports Med 2008;38:425-35.

40. Trivax JE, Franklin BA, Goldstein JA, Chinnaiyan KM, Gallagher MJ, deJong AT, et al. Acute cardiac effects of marathon running. J Appl Physiol 2010;108:1148-53.

41. Laufer EM, Mingels AM, Winkens MH, Joosen IA, Schellings MW, Leiner T, et al. The extent of coronary atherosclerosis is associated with increasing circulating levels of high sensitive cardiac troponin T. Arterioscler Thromb Vasc Biol 2010;30: 1269-75.

42. Omland T, de Lemos JA, Sabatine MS, Christophi CA, Rice MM, Jablonski KA, et al. A sensitive cardiac troponin T assay in stable coronary artery disease. N Engl J Med 2009;361: 2538-47.

43. Wu AH, Jaffe AS, Apple FS, Jesse RL, Francis GL, Morrow DA, et al. National Academy of Clinical Biochemistry Laboratory medicine practice guidelines: use of cardiac troponin and B-type natriuretic peptide or N-terminal proB-type natriuretic peptide for etiologies other than acute coronary syndromes and heart failure. Clin Chem 2007;53:2086-96.

44. Giannitsis E, Becker M, Kurz K, Hess G, Zdunek D, Katus HA. High-sensitivity cardiac troponin $T$ for early prediction of evolving non-ST-segment elevation myocardial infarction in patients with suspected acute coronary syndrome and negative troponin results on admission. Clin Chem 2010;56:642-50.

45. Wu AH, Lu QA, Todd J, Moecks J, Wians F. Short- and long-term biological variation in cardiac troponin I measured with a high-sensitivity assay: implications for clinical practice. Clin Chem 2009;55:52-8.

46. Agarwal SK, Avery CL, Ballantyne CM, Catellier D, Nambi V, Saunders J, et al. Sources of variability in measurements of cardiac troponin $\mathrm{T}$ in a community-based sample: the atherosclerosis risk in communities study. Clin Chem 2011;57:891-7.

47. Vasile VC, Saenger AK, Kroning JM, Jaffe AS. Biological and analytical variability of a novel high-sensitivity cardiac troponin T assay. Clin Chem 2010;56:1086-90.

48. Frankenstein L, Wu AH, Hallermayer K, Wians FH, Giannitsis E, Katus HA. Biological variation and reference change value of high-sensitivity troponin $\mathrm{t}$ in healthy individuals during short and intermediate follow-up periods. Clin Chem 2011;57:706871.

49. Aldous SJ, Florkowski CM, Crozier IG, Elliott J, George P, Lainchbury JG, et al. Comparison of high sensitivity and contemporary troponin assays for the early detection of acute myocardial infarction in the emergency department. Ann Clin Biochem 2011;48:241-8.

50. Jaffe AS. The 10 commandments of troponin, with special reference to high sensitivity assays. Heart 2011;97:940-6.

51. Kassirer JP. Teaching clinical reasoning: case-based and coached. Acad Med 2010;85:1118-24.

52. Bohan JS, Gore JM. Diagnosing acute coronary syndrome: the troponin conundrum. J Watch Emerg Med August 5, 2010 (Accessed on August 16 "www.jwatch.org'),

53. Mehta SR, Granger CB, Boden WE, Steg PG, Bassand JP, Faxon DP, et al. Early versus delayed invasive intervention in acute coronary syndromes. N Engl J Med 2009;360:2165-75.

54. de Winter RJ, Windhausen F, Cornel JH, Dunselman PH, Janus $\mathrm{CL}$, Bendermacher PE, et al. Early invasive versus selectively invasive management for acute coronary syndromes. N Engl J Med 2005;353:1095-104.

55. Melanson SE, Morrow DA, Jarolim P. Earlier detection of myocardial injury in a preliminary evaluation using a new troponin I assay with improved sensitivity. Am J Clin Pathol 2007;128: 282-6.

56. Dolci A, Braga F, Valente C, Guzzetti S, Panteghini M. Impact of implementation of the high-sensitivity cardiac troponin $\mathrm{T}$ assay in a university hospital setting. Clin Chem 2011;57: $1211-2$.

57. Apple FS, Pearce LA, Smith SW, Kaczmarek JM, Murakami MM. Role of monitoring changes in sensitive cardiac troponin I assay results for early diagnosis of myocardial infarction and prediction of risk of adverse events. Clin Chem 2009;55:930-7.

58. Hamm CW, Goldmann BU, Heeschen C, Kreymann G, Berger $\mathrm{J}$, Meinertz T. Emergency room triage of patients with acute chest pain by means of rapid testing for cardiac troponin $\mathrm{T}$ or troponin I. N Engl J Med 1997;337:1648-53.

59. Alpert JS, Thygesen K, Antman E, Bassand JP. Myocardial infarction redefined - a consensus document of The Joint European Society of Cardiology/American College of Cardiology Committee for the redefinition of myocardial infarction. J Am Coll Cardiol 2000;36:959-69.

60. Diamond GA, Kaul S. How would the Reverend Bayes interpret high-sensitivity troponin? Circulation 2010;121:1172-5.

61. Jesse RL. On the relative value of an assay versus that of a test: a history of troponin for the diagnosis of myocardial infarction. J Am Coll Cardiol 2010;55:2125-8.

62. Hess EP, Agarwal D, Chandra S, Murad MH, Erwin PJ, Hollander JE, et al. Diagnostic accuracy of the TIMI risk score in patients with chest pain in the emergency department: a metaanalysis. CMAJ 2010;182:1039-44.

63. Chandra A, Lindsell CJ, Limkakeng A, Diercks DB, Hoekstra JW, Hollander JE, et al. Emergency physician high pretest probability for acute coronary syndrome correlates with adverse cardiovascular outcomes. Acad Emerg Med 2009;16:740-8.

64. Collinson PO, Stubbs PJ. Are troponins confusing? Heart 2003;89:1285-7. 